УДК 532.516

\title{
ТЕПЛООБМЕН И ГИДРОДИНАМИКА В РАДИАЛЬНО-ВРАЩАЮЩЕЙСЯ КРУГЛОЙ ТРУБЕ С НАКЛОННО-ТАНГЕНЦИАЛЬНОЙ ЗАКРУТКОЙ ПОТОКА НА ВХОДЕ
}

Халатов А.А. ${ }^{1,3}$, академик НАН Украины, Кобзарь С.Г. ${ }^{1}$, канд. тех. наук, Дашевский Ю.Я. ${ }^{2}$, канд. тех. наук

${ }^{1}$ Институт технической теплофизики НАН Украины, ул. Желябова, 2а, Киев, 03680, Украина

${ }^{2}$ ГП НПКГ «Зоря»-«Машпроект», пр. Октябрьский, 42a, Николаев, 54018, Украина

${ }^{3}$ Национальный технический университет Украины «КПИ», Проспект Победы 37, Киев, 03056, Украина

В межах зміни параметрів $\mathrm{Ro}=0 \ldots 0,0167$ та $\mathrm{Re}=118000$ виконано чисельне дослідження теплообміну та гідродинаміки у круглій трубі, що радіально обертається, з похило-тангенційним завихренням потоку на вході та $90^{0}$ поворотом на виході. Отримано, що обертання каналу знижує загальний гідродинамічний опір каналу. Обертання каналу в бік завихрення потоку має незначні переваги по теплообміну та опору в порівнянні 3 обертанням проти завихрення потоку, що повинно в враховуватися при проектуванні системи охолодження.

Библ. 6, табл. 5, рис. 9.
В диапазоне изменения параметров $\mathrm{Ro}=0 \ldots 0,0167$ и $\mathrm{Re}=118000$ выполнено численное исследование теплообмена и гидродинамики в радиально-вращающейся круглой трубе с наклонно-тангенциальной закруткой потока на входе и $90^{\circ}$ поворотом на выходе. Получено, что вращение канала снижает общее гидравлическое сопротивление канала. Вращение канала оказывает незначительное влияние на теплоотдачу и распределение локального коэффициента теплоотдачи. Вращение канала в сторону закрутки потока имеет незначительные преимущества по теплообмену и сопротивлению по сравнению с вращением канала против закрутки потока, что должно учитываться при проектировании системы охлаждения.
In the range of parameters $\mathrm{Ro}=0 \quad \ldots 0.0167$ and $\mathrm{Re}=118000$ the numerical investigation of heat transfer and hydrodynamics was performed in rotating radial round tube with incline-tangential flow swirl at the inlet and flow $90^{\circ}$ turn at the exit. It was found that the rotation of the channel reduces the overall flow resistance of the channel. Rotation of the channel towards the flow swirl has a slight advantage on heat transfer and flow resistance compared with the rotation of the channel against the flow swirl, that should be considered when designing a cooling system.

Ключевые слова: : гидродинамика, закрученный поток, теплоотдача, наклонно-тангенциальная закрутка, модели турбулентности.

$d$ - диаметр цилиндрического канала, м;

$f=\frac{\Delta P^{*}}{\Delta x} d \frac{2}{\rho w_{c p}^{2}}-$ коэффициент гидравлического сопротивления;

$n$ - скорость вращения, об./мин;

$P$ - статическое давление, Па;

$P^{*}$ - полное давление, Па;

$T$ - температура, ${ }^{\circ} \mathrm{C}$;

$u, v, w$ - компоненты скорости потока по осям $x$, $y, z, \mathrm{M} / \mathrm{c}$;

\footnotetext{
Введение

Закрученные течения широко применяются в различных технических устройствах для
}

$x, y, z$ - оси декартовой системы координат;

$\alpha-$ коэффициент теплоотдачи, Вт/м² К;

$\varphi-$ угол закрутки потока, градус;

$\Omega$ - угловая скорость вращения, радиан/с;

$v$ - кинематический коэффициент вязкости, $\mathrm{M}^{2} / \mathrm{c}$;

$\rho-$ плотность, кг/ $\mathbf{M}^{3}$;

$\mathrm{Nu}$ - число Нуссельта;

$R e$ - число Рейнольдса;

$\mathrm{Ro}=\frac{\Omega d}{w}-$ параметр вращения.

интенсификации теплообмена и совершенствования различных теплофизических процессов. Подавляющее большинство опубликованных 
исследований выполнены для условий классической тангенциальной закрутки потока, когда поток на входе в трубу подается под углом $90^{\circ}$ к ее продольной оси [1]. В последние годы в связи с разработкой и исследованием внутреннего циклонного охлаждения лопаток газовых турбин самостоятельный интерес получила наклоннотангенциальная закрутка, когда поток подается тангенциально и под некоторым углом менее $90^{\circ}$ к оси канала в направлении движения потока. Большой цикл экспериментальных исследований теплообмена и гидродинамики в этом направлении представлен в работе [2]. Однако эти результаты относятся только к стационарным условиям.

В работе [3] с использованием пакета прикладных программ ANSYS CFX выполнено компьютерное моделирование гидродинамики и теплообмена в круглой трубе с наклоннотангенциальной закруткой потока на входе и $90^{0}$ поворотом потока на выходе, что соответствует условиям системы внутреннего циклонного охлаждения рабочей лопатки газовой турбины. Выполнена верификация двух групп моделей турбулентности, определены основные параметры расчетной сетки, позволяющие получить приемлемую точность расчета. При использовании закрученного потока применительно к системе охлаждения рабочих лопаток газовых турбин большой интерес представляет влияние частоты и направления вращения канала на гидродинамику и теплообмен по длине охлаждающего канала. Экспериментальные работы по данному вопросу в связи со сложностью исследования отсутствуют, пока единственной работой, в которой изучено влияние вращения на теплообмен в круглом канале с тангенциальной $\left(90^{\circ}\right)$ закруткой потока, остается работа [4]. В связи с этим, для исследования влияния вращения на гидродинамику и теплообмен закрученного потока в канале предпочтительно использовать методы компьютерного (CFD) моделирования с привлечением коммерческих пакетов прикладных программ.

Цель и задачи исследования. При геометрических и граничных условиях, характерных для современных рабочих лопаток газовых турбин, с использованием коммерческого программно- го комплекса ANSYS CFX выполнить исследование гидродинамики и теплообмена в радиальновращающейся круглой трубе с наклонно-тангенциальной закруткой потока на входе и $90^{\circ}$ поворотом на выходе для абсолютных значений граничных условий наиболее характерных для современных газотурбинных установок.

Объект исследования и компьютерная модель. В качестве объекта исследований использован круглый канал, который исследовался экспериментально в работах [2,3]. Для соответствия реальным размерам современных лопаток, все размеры канала уменьшены в 5 раз. Объект исследования и его компьютерная модель, построенная в среде ANSYS Design Manager, представлены на рис. 1.

Для выполнения расчетов при вращении канала объект исследования располагался на радиусе вращения $r=405$ мм. Число оборотов задавалось равным 5000, 9500 и 12000 об/мин, которое характерно для современных турбомашин. Исследовалось как правостороннее, так и левостороннее вращение канала. При этом правостороннее вращение направлению в ту же сторону, что и закрутка потока в канале (стрелка на рис. 1, б). При компьютерном моделировании правосторонее вращение канала задавалось положительным значением числа оборотов, а левостороннее отрицательным.

Расчетная сетка построена исходя из параметров, которые показали наилучшие результаты при проведении верификационных расчетовна модельной задаче и отражены в рекомендациях по построению сетки в работе [3]. Параметры расчетной сетки приведены в таблице 1. В качестве модели турбулентности было принята $L R R$ модель напряжений Рейнольдса, которая показала наилучшие результаты по большинству параметров при проведении верификации [3].

Физическая модель была принята аналогичной работе [3]. Граничные условия задавались исходя из реальных параметров современных рабочих лопаток. Расход воздуха составлял $\mathrm{G}=13$ г/с; температура воздуха на входе в объект исследования $\mathrm{T}_{\text {in }}-500{ }^{\circ} \mathrm{C}$; температура стенки завихрителя $\mathrm{T}_{\mathrm{wz}}-720{ }^{\circ} \mathrm{C}$; 


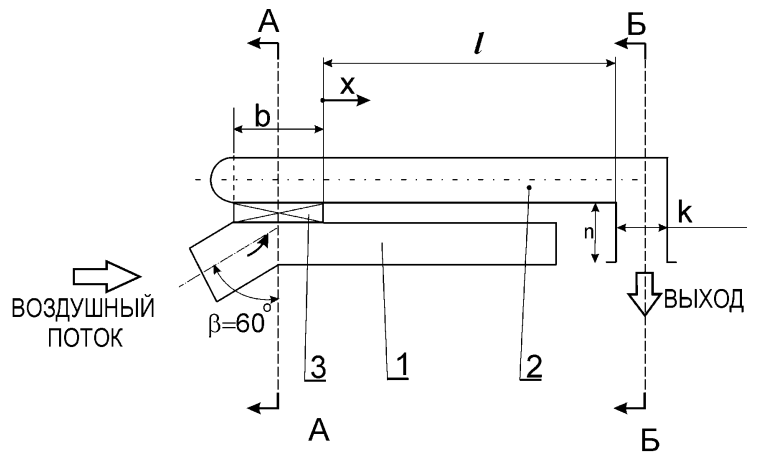

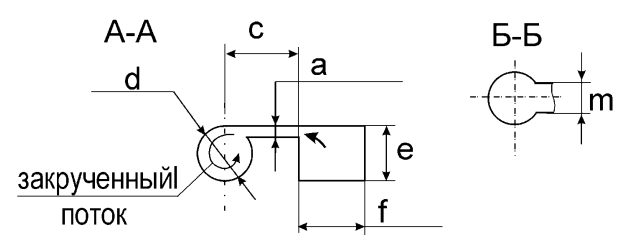

a)

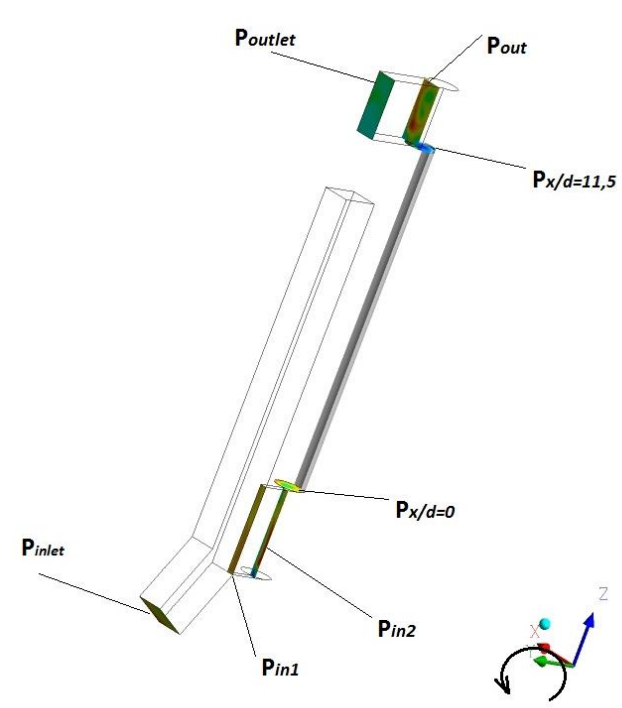

б)

Рис. 1. Геометрическая модель объекта исследования (а) [1] и его компьютерная модель (б): 1 -распределительный канал; 2 - тангенциальный завихритель; 3 -основной цилиндрический

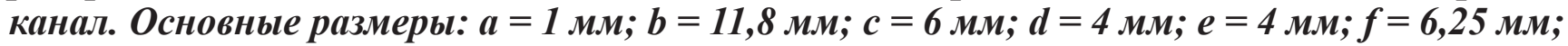
$k=8 \mathrm{Mм} ; l=46,5 \mathrm{MM} ; m=3 \mathrm{MM} ; n=8 \mathrm{MM}$.

Таблица 1. Параметры расчетной сетки

\begin{tabular}{|l|l|c|}
\hline \multirow{2}{*}{ Сгущение } & \multicolumn{2}{|c|}{$1,0 \mathrm{e}^{-4}$} \\
\cline { 2 - 3 } & Максимальная толщина слоя, м & 15 \\
\cline { 2 - 3 } & Количество слоев, шт. & 1,2 \\
\cline { 2 - 3 } & Фактор роста & $4 \mathrm{e}^{-4}$ \\
\hline Максимальный размер ячейки на поверхностях, м & $4 \mathrm{e}^{-4}$ \\
\hline Максимальный размер ячейки, м & 808325 \\
\hline Количество элементов, шт & 1694651 \\
\hline Количество узлов, шт & \\
\hline
\end{tabular}

температура стенки цилиндрического канала $\mathrm{T}_{\mathrm{wz}}-720^{\circ} \mathrm{C}$.

На выходе из расчетной области задавалось осредненное статическое давление равное 750000 Па. При данных граничных условиях среднее по длине канала значение числа Рейнольдса составляло 118000. Значение полного давления на входе в модель определялось в процессе расчета.

\section{Гидродинамика закрученного потока}

Изменение поверхностного угла закрутки потока по длине канала в зависимости от скорости и направления вращения показано на рис. 2 и 3. Координата $x$ отсчитывается от «среза» тангенциальной щели завихрителя. Во всех случаях продольное распределение угла закрутки имеет «острый» максимум, который расположен в области $x / d \approx 0,5$. Это связано с тем, что в промежутке $0,5<x / d<1,0$ формируется перестройка потока от условий подачи потока в канал до закономерностей течения закрученного потока в круглом канале. Далее по длине канала закрутка потока снижается более монотонно. Влияние вращения канала на закрутку потока проявляется двояко. В начале канала существует зона, где с увеличением скорости вращения происходит снижение поверхностного угла закрутки потока. В конце канала увеличение скорости вращения даже увеличивает закрутку потока.

Для правостороннего вращения измене- 
ния характера влияния скорости вращения на закрутку потока соответствует сечению $x / d \approx 6,8$, а для левостороннего вращения $x / d \approx 4,5$. Следует отметить, что левостороннее вращение канала более заметно влияет на закрутку потока в канале, чем правостороннее. В частности, при левостороннем вращении канала на начальном участке наблюдается значительное снижение максимального значения поверхностного угла закрутки с увеличением скорости вращения.

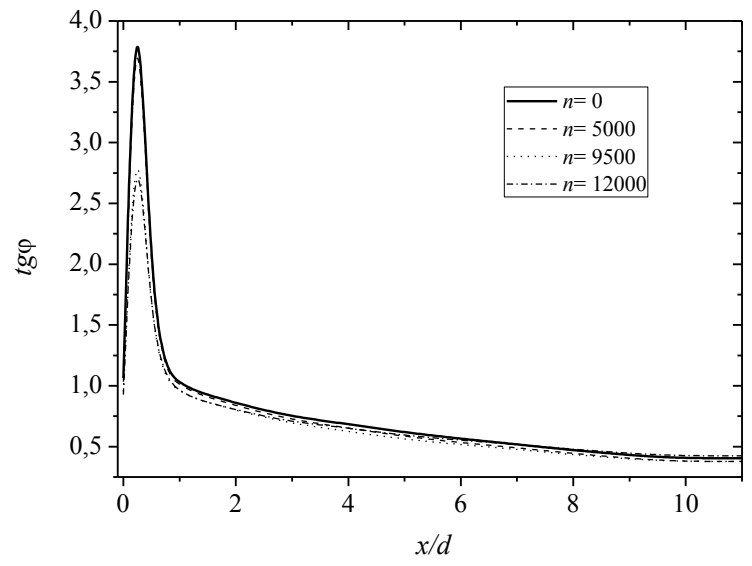

Рис. 2. Изменение поверхностного угла закрутки потока по длине канала при правостороннем вращении.

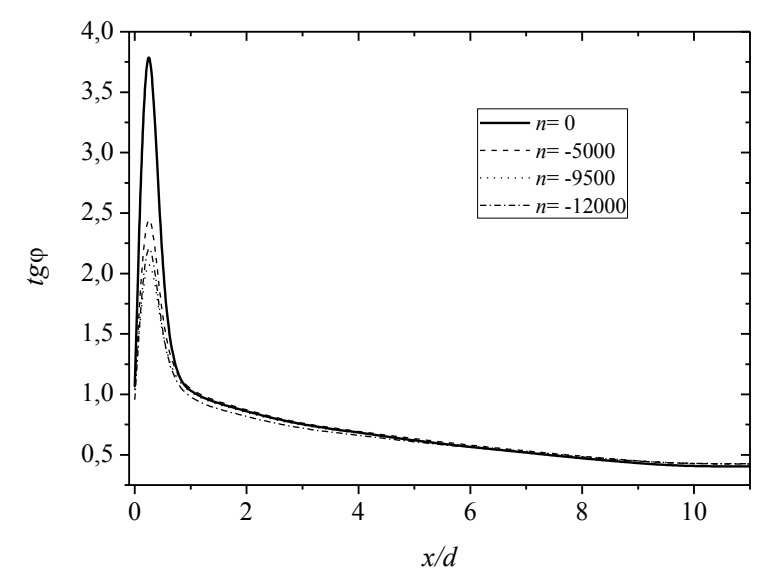

Рис. 3. Изменение поверхностного угла закрутки потока по длине канала при левостороннем вращении.

Подтверждением того, что в промежутке $0,5<x / d<1,0$ происходит быстрая перестройка структуры потока после выхода из канала завихрителя служат результаты, полученные для продольного распределения коэффициента гидравлического сопротивления канала, которые представлены на рис. 4 и 5 . В сечении $x / d \approx 1,0$ линия поведения коэффициента сопротивления имеет незначительный перегиб. Если до этой точки коэффициент сопротивления имеет резкое изменение, то после сечения $x / d \approx 1,0$ характер его изменения становится более плавным.

Влияние скорости вращения на коэффициент сопротивления начинает сказываться, начиная с сечения $x / d \approx 0,5$, и приводит к его уменьшению. При частоте вращения 12000 об/мин в конце канала в сечении $x / d \approx 9,75$ наблюдается незначительное отрицательное значение коэффициента трения. Это, скорее всего, связано с геометрическими особенностями объекта исследования, а именно наличием поворота на $90^{\circ}$ на выходе из канала. Небольшое увеличение полного давления в конце канала при больших скоростях вращения, скорее всего, связано с действием центробежных сил на поток, что требует дополнительного изучения.

В целом, вращение канала способствует уменьшению гидравлических потерь в канале, что может быть объяснено «насосным» эффектом, который возникает в радиально-вращающихся каналах. Заметное снижение потерь наблюдается при частоте оборотов $n>9500$ об/мин.

Распределения полного и статического давлений по длине канала при правостороннем вращении канала показаны на рис. 6 и 7. Изменение полного давления в характерных точках исследованной геометрии (рис. 1, б) представлены в таблице 2.

Вращение снижает затраты полного давления на прокачку потока в модели. Изменение полного и статического давления по длине канала при левостороннем вращении имеет похожий характер. Результаты определения полного давления в характерных точках исследованной геометрии, показали, что в стационарных условиях для обеспечения прокачки расхода $\mathrm{G}=13$ г/с воздуха, на входе в исследуемую геометрию требуется величина полного давления равная 1375590 Па. При вращении потребное для прокачки этого расхода значение полного давления на входе в объект исследования уменьшается. При этом правостороннее вращение модели обеспечивает большее сни- 


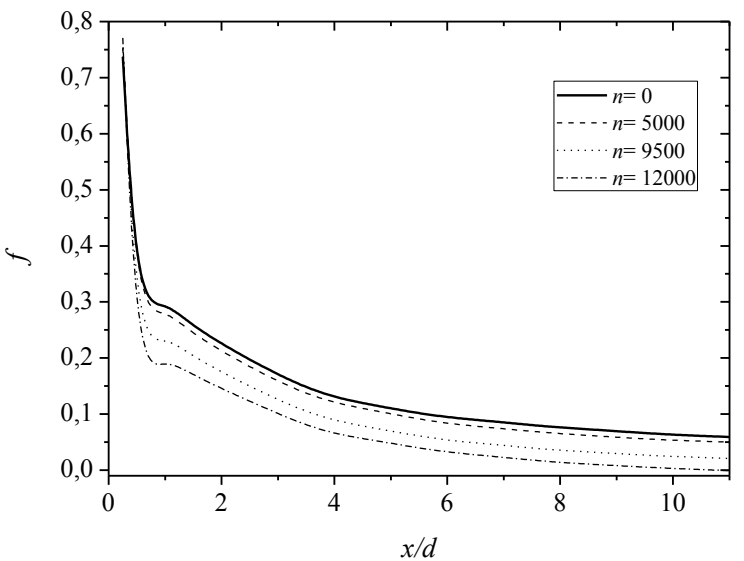

Рис. 4. Продольное изменение коэффициента гидравлического сопротивления канала при правостороннем вращении.

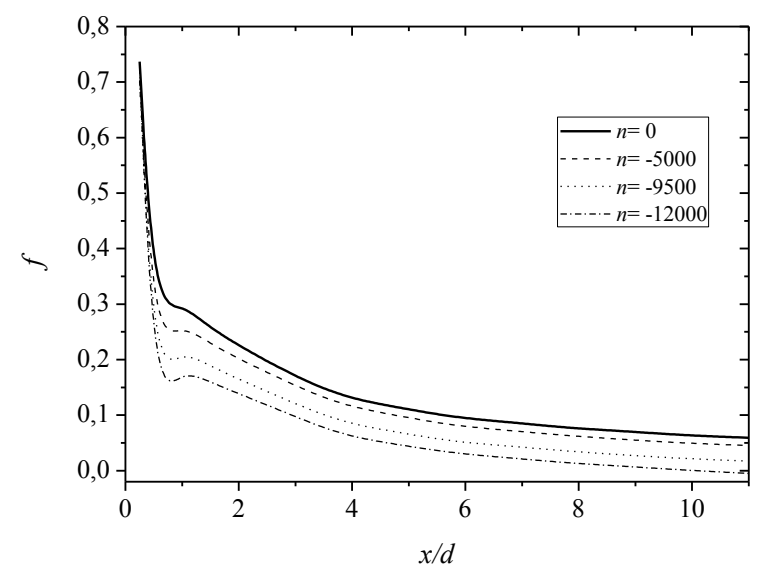

Рис. 5. Продольное изменение коэффициента гидравлического сопротивления канала при левостороннем вращении.

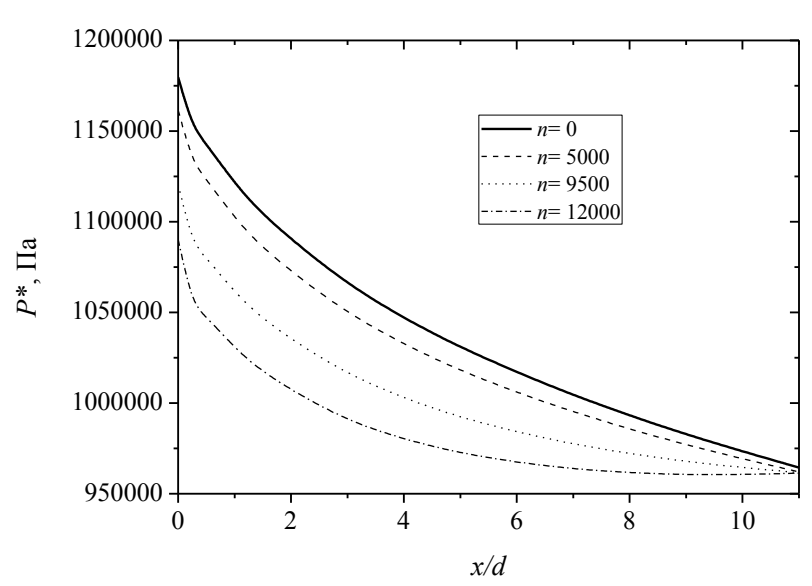

Рис. 6. Продольное изменение полного давления в канале при правостороннем вращении.

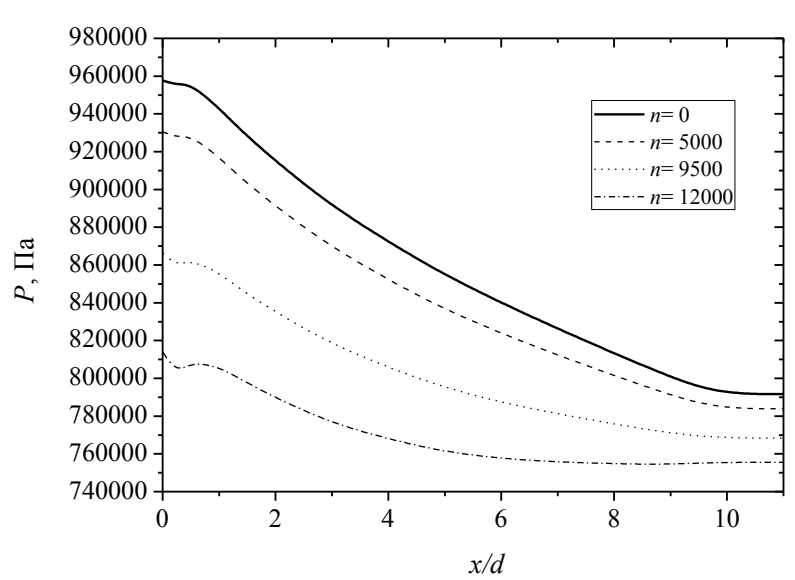

Рис. 7. Продольное изменение статического давления на стенке канала при правостороннем вращении.

Таблица 2. Значения полного давления в характерных точках канала при его вращении

\begin{tabular}{|c|c|c|c|c|c|c|c|}
\hline$n$, об/мин & 0 & 5000 & 9500 & 12000 & -5000 & -9500 & -12000 \\
\hline$P^{*}{ }_{\text {inlep }}$ Па & 1375590 & 1362330 & 1295270 & 1247060 & 1347080 & 1281120 & 1246500 \\
\hline$P^{*}{ }_{i n l}$, Па & 1326900 & 1321430 & 1273300 & 1238570 & 1304760 & 1256320 & 1234750 \\
\hline$P^{*}{ }_{\text {in } 2}$, Па & 1307300 & 1301320 & 1252300 & 1216350 & 1284790 & 1234760 & 1212510 \\
\hline$P_{x / d=0}^{*}$, Па & 1191350 & 1184200 & 1135990 & 1098440 & 1164790 & 1115940 & 1087180 \\
\hline$P^{*}{ }_{x / d=11,5}, \Pi а$ & 964680 & 969427 & 967534 & 965119 & 964412 & 961899 & 966516 \\
\hline$P^{*}{ }_{\text {oup }}$ Па & 913287 & 916899 & 919661 & 919230 & 914159 & 915830 & 920766 \\
\hline$P_{\text {ouflet }}^{*}$ Па & 838990 & 841998 & 844129 & 844897 & 841742 & 843127 & 841726 \\
\hline
\end{tabular}


жение давления, по сравнению с левосторонним вращением, что связано с благоприятным влиянием Кориолисовой силы на поток при выходе из завихрителя.

Влияние вращения канала на структуру закрученного потока при характерных для рабочих лопаток параметрах изучено мало. В открытой печати не удалось обнаружить экспериментальных и теоретических результатов, которые имели бы непосредственное отношение к рассматриваемой задаче. В работах $[5,6]$ приведена методика определения перепада полного давления в радиально-вращающемся канале для более простого случая, когда вынужденное течение отсутствует. Согласно приведенной методики, перепад давления в канале может быть рассчитан по формуле:
$\Delta p=p_{2}-p_{1}=p_{1}\left(\exp \frac{\omega^{2}\left(r_{2}^{2}-r_{1}^{2}\right)}{2 R T}-1\right)$,

где $R$ - газовая постоянная охлаждающего воздуxa.

По формуле (1) была выполнена оценка перепада полного давления в цилиндрическом канале. Начало канала находится на радиусе $r_{l}=$ 0,4178 м, а его выход - на радиусе $r_{2}=0,4638 \mathrm{м}$. Температура охлаждающего воздуха на входе в канал - 773 К. Результаты сравнения перепада давления, обусловленного вращением исследуемого канала и перепада давления в таком же канале, рассчитанного по формуле (1) приведены в таблице 3.

Результаты, приведенные в таблице, показывают, что при вращении закрученного потока

Таблица 3. Перепад полного давления в канале в зависимости от числа оборотов

\begin{tabular}{|c|c|c|c|c|}
\hline$n$, об/мин & 0 & 5000 & 9500 & 12000 \\
\hline$\omega$, радиан/с & 0 & 523 & 988 & 1248 \\
\hline P1, Па & 1191350 & 1184200 & 1135990 & 1098440 \\
\hline Р2, Па & 964680 & 969427 & 967534 & 965119 \\
\hline P1 - P2, Па & 226670 & 214773 & 168456 & 133321 \\
\hline$\Delta \mathrm{P}, \Pi \mathrm{a}$ & 0 & 11897 & 58214 & 93349 \\
\hline $\begin{array}{l}\Delta \mathrm{P}, \text { Па, } \\
\text { формула (1) }\end{array}$ & 0 & 40840 & 111882 & 140811 \\
\hline
\end{tabular}

в цилиндрическом канале перепад полного давления ниже, чем при отсутствии вынужденного движения. Данный факт, прежде всего, связан с потерями полного давления за счет гидравлических потерь при движении закрученного потока в канале.

Характер изменения полного давления в контрольных сечениях (табл. 2) показывает, что основной источник потерь полного давления находится в завихрителе, в котором имеют место потери на внезапное сужение, внезапное расширение и поворот потока. Значения рассчитанных местных потерь в завихрителе и в повороте, в зависимости от частоты вращения, представлены в таблице 4.

Коэффициент гидравлического сопротивления завихрителя определялся соотношением:

$$
\zeta_{z}^{*}=\frac{\Delta P^{*}}{\rho_{u} \frac{\bar{w}_{u}{ }^{2}}{2}}
$$

где $\Delta P^{*}$ - разность полных давлений перед завихрителем и в сечении трубы за завихрителем на расстоянии $x / d=0$, где координата $x$ отсчитывается от «среза» тангенциальной щели завихрителя; $w_{u}, \rho_{u}-$ среднемассовая скорость и плотность воздуха в щели завихрителя.

Коэффициент гидравлического сопротивления выходного поворота определялся по соотношению:

$\zeta_{\text {turn }}^{*}=\frac{\Delta P^{*}{ }_{t u r n}}{\rho_{c p} \frac{w_{c p}{ }^{2}}{2}}$

где $\Delta P_{\text {turn }}^{*}-$ разность полных давлений перед 
Таблица 4. Потери полного давления в наклонно-тангенциальном завихрителе и в повороте

\begin{tabular}{|c|c|c|c|c|c|c|c|}
\hline$n$, об/мин & 0 & 5000 & 9500 & 12000 & -5000 & -9500 & -12000 \\
\hline$\zeta_{\mathrm{z}}^{*}$ & 2,614 & 2,514 & 2,192 & 2,0 & 2,49 & 2,296 & 0,201 \\
\hline$\zeta_{\text {turn }}^{*}$ & 0,752 & 0,7407 & 0,704 & 0,671 & 0,732 & 0,762 & 0,672 \\
\hline
\end{tabular}

поворотом и после него; $w_{c p}, \rho_{c p}-$ среднемассовая скорость потока в канале и плотность в цилиндрическом канале на входе в поворот.

Результаты расчетов показали, что вращение уменьшает потери в завихрителе и в повороте. Правостороннее вращение имеет несколько больший эффект снижения, по сравнению с левосторонним вращением.

\section{Теплообмен закрученного потока}

Результаты расчета теплоотдачи во вращающемся канале представлены на рис. 8 и 9. Как следует, распределение числа Нуссельта имеет максимум в районе $x / d \approx 0,5$, что соответствует сечению, где наблюдается максимум поверхностного угла закрутки потока (рис. 2, 3). После максимума происходит постепенное уменьшение числа Нуссельта вплоть до конца трубы. В целом вращение оказывает слабое влияние на теплоотдачу.

При правостороннем вращении увеличение числа оборотов приводит к незначительному увеличению теплоотдачи по всей длине канала. Благоприятное воздействие на поток силы Кориолиса приводит к некоторому росту теплоотдачи при увеличении скорости вращения, что следует из данных таблицы 5. Однако эта интенсификация происходит только на участке канала $0<x / d$ $<1,0$ и не превосходит $3,6 \%$. Средняя по длине канала интенсификация теплоотдачи $(\mathrm{Ro}=0,0167)$ составляет $1,8 \%$ (табл. 5).
При левостороннем вращении сила Кориолиса оказывает отрицательное воздействие на поток - она снижает теплоотдачу в канале. C увеличением числа оборотов снижение теплоотдачи более существенно проявляется в начале канала (до $x / d<1,0$ ), где средняя теплоотдача снижается на 2,4\% $(\mathrm{Ro}=0,0167)$. При этом уменьшение среднего числа Нуссельта по всей длине канала составляет всего 1,5 \% (табл. 5).

Полученные результаты по влиянию вращения канала на теплоотдачу качественно согласуются с данными работы [4], в которой выполнено исследование теплообмена в радиально-вращающемся канале с закрученным внутренним течением. Исследования были выполнены при $\mathrm{Re}_{d}$ $=20000$ и Ro $=0 \ldots 0,023$. Было показано, что правостороннее вращение канала способствует увеличению среднего по длине канала числа Нуссельта приблизительно на 9 \% по сравнению со стационарным случаем, что объясняется положительным влиянием силы Кориолиса. При левостороннем вращении наблюдалось снижение теплоотдачи.

В наших исследованиях при значении параметра вращения Ro $=0,0167$, увеличение средней теплоотдачи составляет всего 1,8 \%. Данный результат может быть объяснен условиями закрутки потока в канале и различием в их геометрии [4]. В работе [4] подвод охлаждающего воздуха осуществлялся через систему дискретных

Таблица 5. Среднее число Нуссельта в канале

\begin{tabular}{|c|c|c|c|c|c|c|c|}
\hline n, об/мин & 0 & 5000 & 9500 & 12000 & -5000 & -9500 & -12000 \\
\hline Rо & 0 & 0,007 & 0,013 & 0,0167 & 0,007 & 0,013 & 0,0167 \\
\hline $\begin{array}{l}\text { Число } \overline{\mathrm{Nu}}, \\
x / d=0 \ldots 1,0\end{array}$ & 529,83 & 537,54 & 548,34 & 549,0 & 519,0 & 517,28 & 517,0 \\
\hline $\begin{array}{l}\text { Число } \overline{\mathrm{Nu}}, \\
\text { /d }=1,0 . .11,0\end{array}$ & 434,43 & 441,08 & 441,1 & 441,45 & 430,8 & 429,37 & 428,344 \\
\hline $\begin{array}{l}\text { Число } \overline{\mathrm{Nu}}, \\
x / d=0 . .11,0\end{array}$ & 442,68 & 449,42 & 450,12 & 450,67 & 438,42 & 436,99 & 436,04 \\
\hline
\end{tabular}




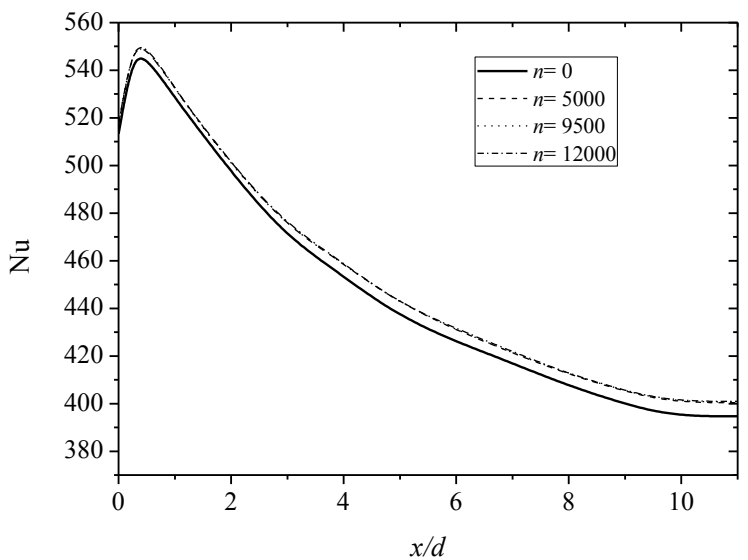

Рис. 8. Продольное изменение локального числа Нуссельта в канале при правостороннем вращении канала.

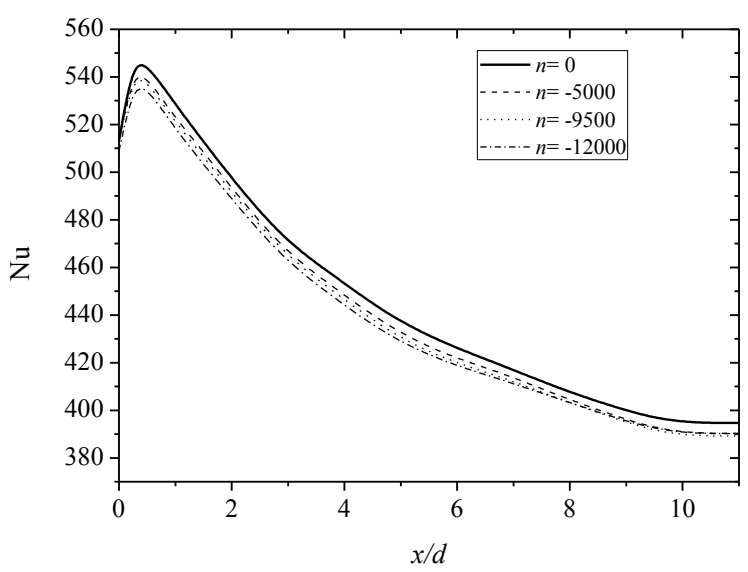

Рис. 9. Продольное изменение локального числа Нуссельта в канале при левостороннем вращении канала.

тангенциальных завихрителей $\left(90^{\circ}\right)$, равномерно расположенных по длине круглого канала, таким образом, поток охладителя в цилиндрический канал подавался множественными струями. Значение числа Рейнольдса в нашем исследовании было в шесть раз выше, чем в работе [4], что определяет более значительное воздействие центробежной силы.

\section{Выводы}

$\mathrm{B}$ области $\mathrm{Ro}=0 \ldots 0,0167$ и $\mathrm{Re}=118000$ выполнено численное исследование теплообмена и гидродинамики в радиально-вращающейся круглой трубе с наклонно-тангенциальной закруткой потока на входе и $90^{\circ}$ поворотом на выходе. Полученные результаты позволяют сделать сле- дующие выводы:

1. Вращение канала снижает общее гидравлическое сопротивление канала.

2. С увеличением частоты вращения местные гидравлические потери в области завихрителя и в выходном повороте снижаются.

3. Вращение канала оказывает незначительное влияние на теплоотдачу и распределение локального коэффициента теплоотдачи.

4. Вращение канала в сторону закрутки потока (правостороннее вращение) имеет незначительные преимущества по теплообмену и сопротивлению по сравнению с вращением канала против закрутки потока (левостороннее вращение), что должно учитываться при проектировании системы охлаждения.

5. Учитывая полученные результаты, дальнейшее исследование должно быть, направлено на изучение влияния Кориолисовой силы на теплообмен и сопротивление в канале для рассматриваемого типа закрутки потока.

\section{ЛИТЕРАТУРА}

1. Теплообмен и гидродинамика в полях центробежных массовых сил. Т.5. «Тепломассобмен и теплогидравлическая эффективность вихревых и закрученных потоков» / Халатов А.А., Борисов И.И., Шевцов С.В./ Киев: Изд. Ин-та технической теплофизики НАНУ. - 2005. - 500с.

2. Теплообмен и гидродинамика в полях центробежных массовых сил. Т.9. «Теплообмен и гидродинамика при циклонном охлаждении лопаток газовых турбин» / Халатов А.А., Романов В.В., Борисов И.И., Дашевский Ю.Я., Северин С.Д. / Киев: Изд. Ин-та технической теплофизики НАНУ. - 2010.-317c.

3. Халатов А.А., Кобзарь С.Г., Дашевский Ю.Я. Численное моделирование теплообмена и гидродинамики в круглой трубе с наклонно-тангенциальной закруткой потока на входе // Промышленная теплотехника. - 2015.- Т.37, №1. C.12-21.

4. Glezer B., Moon H.-K., Kerrebrock J., Bons $J$., Guenette $G$. Heat transfer in rotating radial channel with swirling internal flow // ASME Paper 98-GT-214.

5. Копелев С.3., Гуров С.В. Тепловое состоя- 
ние элементов конструкции авиационных двигателей. М.: Машиностроение. - 1978. - 208c.

6. Теплопередача в охлаждаемых деталях га- зотурбинных двигателей / В.И. Локай, М.Н. Бодунов, В.В. Жуйков, А.В. Щукин. 2-е изд., перераб. и доп. - М.: Машиностроение, - 1993. - 288c.
THE HEAT TRANSFER AND HYDRODYNAMICS IN ROTATING RADIAL ROUND TUBE WITH INCLINEDTANGENTIAL FLOW SWIRL AT THE INLET

\section{Khalatov A. ${ }^{1,3}$, Kobzar S. ${ }^{1}$, Dashevskyy Yu. ${ }^{2}$}

${ }^{1}$ Institute of Engineering Thermophysics, National Academy of Sciences of Ukraine, 2a Zhelyabova str., Kiev, 03680, Ukraine

${ }^{2} \mathrm{GP} \quad \mathrm{NPKG}$ «Zorya»-Mashproekt», 42 a, Pr. Oktyabrskiy, Nikolev, 54018, Ukraine

${ }^{3}$ The National Technical University «KPI», 37 Pobedi Avenue, Kiev, 03056, Ukraine

In the range of parameters Ro $=0 \ldots 0.0167$ and $\mathrm{Re}=118000$ the numerical investigation of heat transfer and hydrodynamics was performed in rotating radial round tube with incline-tangential flow swirl at the inlet and flow $90^{\circ}$ turn at the exit. It was found that the rotation of the channel reduces the overall flow resistance of the channel. Rotation of the channel towards the flow swirl has a slight advantage on heat transfer and flow resistance compared with the rotation of the channel against the flow swirl, that should be considered when designing a cooling system.

Ref. 6, tab. 5, fig. 9.

Key words: hydrodynamics, swirling flow, heat transfer, inclined-tangential flow swirl, rotation, turbulence models.
1. Heat transfer and fluid flow in centrifugal fields. V.5. «Heat and mass transfer? Thermalhydraulic performance of vortex and swirling flows / Khalatov A.A., Borisov I.I., Shevtsov/ Institute for Engineering Thermophysics. National Academy of Sciences of Ukraine. - Kyiv. - 2005. - 500p. (Rus.)

2. Heat transfer and fluid flow in centrifugal fields. V.9. «Heat transfer and hydrodynamics at cyclone cooling of gas turbine blades» / Khalatov A.A., Romanov V.V., Borisov I.I., Dashevskyy Y.Y., Severin S.D./ Institute for Engineering Thermophysics. National Academy of Sciences of Ukraine. - Kyiv. -2010 . -317p. (Rus.)

3. Khalatov A.A., Kobzar S.G., Dashevskyy Y.Y. Numerical simulation of heat transfer and hydrodynamics in round tube with inclinedtangential flow swirl at the inlet // Promushlennaya teplotekhnyka. - 2015.- v.37, №1.-pp.12-21. (Rus.)

4. Glezer B., Moon H.-K., Kerrebrock J., Bons $J$., Guenette G. Heat transfer in rotating radial channel with swirling internal flow // ASME Paper 98-GT-214.

5. Kopelev S.Z., Gurov S.V. The thermal state of the structural elements of aircraft engines/ Moscow.Mashinostroenie. - 1978. - 208p. (Rus.)

6. Heat transfer in gas turbine engines cooled details / Lokai V.I, Bodunov M.N., Zhuikov V.V., Schukin A.V./ Moscow.- Mashinostroenie. - 1993. -288 p. (Rus.) 